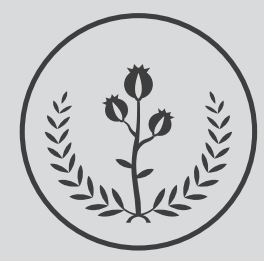

FUCS

\title{
Re

\section{Narrativa de enfermería: cuidado holístico de un paciente con pie diabético}

\section{Nursing narrative: holistic management of diabetic foot}

${ }^{a}$ Enfermería, Cuidado del paciente en urgencias, Fundación Universitaria de Ciencias de la Salud. Bogotá DC, Colombia.

\section{R E S U M E N}

Los enfermeros son profesionales que brindan cuidado y realizan intervenciones en diferentes campos de acción del proceso salud-enfermedad para promover estilos de vida, preservar y mantener la vitalidad de las personas y comunidades y propender por una recuperación y rehabilitación que permita la integración adecuada de los pacientes a una vida productiva y familiar exitosa. En este contexto, quienes cursan con diagnóstico de diabetes mellitus se favorecen con el apoyo de enfermeros que pueden o tienen la capacidad de cubrir sus requerimientos en términos de educación frente a la patología, al control de los factores de riesgo y al manejo adecuado de una complicación principal de esta enfermedad como lo es el pie diabético; por eso esta profesión se convierte en una fuente de educación, cuidado, conocimientos y bienestar no solo para el paciente sino para su contexto familiar.

Palabras clave: enfermería, cuidado, diabetes mellitus, pie diabético, educación, familia, comunicación.

(C) 2021 Fundación Universitaria de Ciencias de la Salud - FUCS. Este es un artículo Open Access bajo la licencia CC BY-NC-ND (http://creativecommons.org/licenses/by-nc-nd/4.0/).

Historia del artículo: Fecha recibido: diciembre 15 de 2020 Fecha aceptado: febrero 19 de 2021

\section{INFORMACIÓN DEL ARTÍCULO}

Autor para correspondencia. Elizabeth Catalina Rincón Ballén ecrincon@fucsalud.edu.co
DOI

10.31260/RepertMedCir.01217273.1156 


\section{A BST R ACT}

Nurses are professionals who provide care interventions in different fields of action of the health-disease process to promote healthy lifestyles, preserve and maintain the individual's and community vitality, and advocate for patients' recovery and rehabilitation allowing their proper and successful integration to productive activities and family life. In this context, those with a diagnosis of diabetes mellitus benefit from the support of nurses who can meet their requirements or have the ability to do so, in terms of educating patients on their condition, to control risk factors and facilitate learning the proper management of diabetic foot, a major complication of diabetes; therefore, these professionals become a source of education, care, knowledge and wellbeing not only for the patient but also for his family context.

Key words: nursing, care, diabetes mellitus, diabetic foot, education, family, communication.

(C) 2021 Fundación Universitaria de Ciencias de la Salud - FUCS This is an open access article under the CC BY-NC-ND license (http://creativecommons.org/licenses/by-nc-nd/4.0/).

\section{INTRODUCCIÓN}

La diabetes mellitus es una enfermedad crónica en la cual se engloban diversos trastornos a nivel metabólico como son la presencia de hiperglucemia, defectos en la secreción/elaboración y acción de la insulina tanto en el páncreas como en el resto del cuerpo y alteraciones en el metabolismo de grasas y proteínas. ${ }^{1}$ Es una enfermedad que genera daño en diversos órganos y sistemas (en especial riñones, ojos, nervios, corazón y vasos sanguíneos) y como complicación provoca la aparición del pie diabético (la más común) presentando diferentes tipos de lesiones y ulceras. El pie diabético implica la perdida de la integridad cutánea afectando desde el epitelio hasta las capas más profundas de las estructuras, las cuales incluso pueden involucrar afectación de los músculos y huesos.

La diabetes se ha configurado como un problema de salud debido a las graves complicaciones que puede generar, los altos costos para su intervención, tratamiento y rehabilitación y la alta demanda en cuanto a recursos físicos y humanos necesarios de los sistemas de salud. ${ }^{2}$ De acuerdo con la Organización Mundial de la Salud (OMS) el número de pacientes diabéticos en 2000 era de 171 millones y según diversos estudios pronósticos se espera que para 2025 haya 380 millones de personas con esta patología. ${ }^{3}$ Esta alta prevalencia, morbilidad y mortalidad en todo el mundo resalta la necesidad de realizar intervenciones educativas tanto en prevención primaria como secundaria, crear planes y programas orientados a la rehabilitación y seguimiento previamente diagnosticados y a la formación de profesionales idóneos que cuenten con conocimientos específicos en el manejo de esta patología y sus respectivas complicaciones (con énfasis en el manejo de pie diabético debido a su relevancia).

La profesión de enfermero juega un papel protagónico en la prevención y el diagnóstico temprano de la diabetes y sus complicaciones. La importancia del rol puede reflejarse en los sistemas de salud, en la educación a nivel comunitario, en el cuidado del paciente tanto a nivel intra como extrahospitalario y en la mejoría de la calidad de vida de las personas y comunidades. Combina ciencia y arte para proveer servicios en salud y busca eliminar o reducir necesidades físicas, emocionales, mentales, socialesculturales y espirituales en los pacientes. ${ }^{4}$

Debido a lo anterior, los enfermeros juegan un papel importante en el reconocimiento temprano de cambios en la piel, a nivel de sensibilidad y percepción distal, en el cuidado de los pies y en la aplicación y utilización de tecnologías emergentes para tratar el pie diabético como la complicación más frecuente de la diabetes. Esta patología es la causa más frecuente de hospitalización en diabéticos ya que puede evolucionar a infecciones, gangrena, amputaciones de extremidades e incluso puede haber fallecimiento si las necesidades en cuanto a cuidado no son atendidas. La enfermería puede brindar planes de cuidado e intervenciones individualizadas que se orienten a mejorar la calidad de vida y a favorecer una rehabilitación, tanto a nivel asistencial como domiciliario, es por ello que el profesional debe comprender la dimensión de esta patología ya que el campo de acción es bastante amplio y los pacientes llegan a favorecerse con todas las acciones y decisiones que realiza.

\section{NARRATIVA DE UNA SITUACIÓN} EN ENFERMERÍA

Me encontraba en el servicio de hospitalización de la clínica preparándome para el recibo de turno cuando mi compañera me comentó sobre el ingreso de un paciente nuevo. En esta área se pueden recibir pacientes de cualquier especialidad y servicio de origen ya sea de urgencias, UCI o salas de cirugía. Yo llevaba más o menos una semana en el piso entonces conocía todos los que se encontraban internados en el momento. 
El paciente mencionado ingresó a la clínica por presentar heridas por arma cortopunzante debido a que fue víctima de robo (el suceso ocurrió precisamente cerca de la clínica). Llegó a urgencias en donde se valoraron dos heridas bilaterales a nivel del tórax por lo cual fue estabilizado y de forma rápida se trasladó a salas de cirugía para su intervención. Allí le colocaron dos tubos a tórax y después fue remitido a la UCI. Cuando el paciente presentó signos de estabilización ingresó a hospitalización para continuar su proceso de recuperación. En el recibo de turno conocí al paciente, un adulto mayor que presentaba los dos tubos de tórax, requería oxigenoterapia y líquidos, tenía como antecedente diabetes por lo cual presentaba además signos de pie diabético ( 2 ulceras pequeñas y superficiales en ambos pies) y disminución de la visión. En ese momento valoré el paciente, establecí una pequeña charla y pude notar que no contaba con familiares o acompañantes presentes. Debido al diagnóstico estuvo cerca de tres semanas en piso, por lo tanto tuve la oportunidad de interactuar con él en varias ocasiones, lo que me permitió concluir que su red familiar estaba ausente la mayor parte del tiempo debido a sus ocupaciones laborales y de otro lado refirió que vivía solo. Manifestó que desde su diagnóstico percibió pérdida gradual de visión de manera considerable lo que le impidió detectar los cambios en la piel de los pies, notando la presencia de heridas cuando le produjeron algún tipo de malestar, incomodidad y dolor. Solo lo visitaban unos vecinos y eran los que estaban más al tanto de su situación. Con el transcurrir del tiempo comenzó a presentar zonas anómalas por presión en el talón de ambos pies, por lo cual se tuvo que hacer mayor valoración e integrar a todos los turnos en la necesidad de cuidado de la piel y en la identificación de los errores que se cometieron para que el paciente deteriorara su integridad cutánea. Cuando ingresó al servicio de hospitalización no se realizó la respectiva notificación a clínica de heridas por lo que esta no tenía en su listado al paciente para realizar inspección e intervenciones específicas y especializadas. Después de la notificación había ocasiones en que el servicio de clínica de heridas no podía atender al paciente, por lo cual se tuvo que realizar mayor intervención entre los turnos para evitar que se deteriorara su estado general. Un aspecto a considerar es que no contaba con una red familiar fuerte, pues no tenía compañía permanente que garantizara una hidratación de la piel o que realizara vigilancia del aspecto cutáneo y además tenía inconvenientes en la movilización ya que tenía que desplazarse con ayuda y en silla de ruedas debido a las lesiones de las zonas plantares, a la presencia de los tubos de tórax y a los líquidos intravenosos que requería. Como no contaba con los elementos necesarios para el cuidado cutáneo como cremas, ungüentos o humectantes se avisó al servicio de seguridad sobre sus condiciones para que hicieran un puente de comunicación con la familia y pudieran conseguir los recursos que necesitaba.

Poco tiempo después se presentaron algunos familiares, se les puso al tanto de la situación actual y se les indicó la importancia y necesidad de que hubiera siempre un acompañante y de tener ciertos elementos de aseo e hidratación vitales para prevenir mayores complicaciones. Ellos se organizaron y pudieron conseguir los recursos y materiales requeridos y se les brindó educación por parte de clínica de heridas como de los enfermeros que estaban en cada turno sobre el diagnóstico, la implicación del pie diabético y los cuidados en el hogar.

\section{ANÁLISIS Y CONTEXTUALIZACIÓN} DE LA SITUACIÓN

Nos encontramos con un paciente que tiene un antecedente de diabetes complicada por pie diabético. Durante el recibo de turno pude valorar las lesiones clasificándolas según se indica en la tabla 1.

Tabla 1. Puntuación y graduación de la herida (San Elian)

\begin{tabular}{|c|c|c|}
\hline \multicolumn{2}{|c|}{ Factores } \\
\hline $\begin{array}{c}\text { Localizacion inicial } \\
\text { Metatarsal (2) }\end{array}$ & $\begin{array}{c}\text { Isquemia } \\
\text { Leve (1) }\end{array}$ & $\begin{array}{c}\text { Profundidad } \\
\text { Parcial (2) }\end{array}$ \\
\hline $\begin{array}{c}\text { Aspecto Topográfico } \\
\text { Plantar (1) }\end{array}$ & $\begin{array}{c}\text { Infección } \\
\text { Leve (1) }\end{array}$ & $\begin{array}{c}\text { Área } \\
\text { Pequeña <10 (1) }\end{array}$ \\
\hline $\begin{array}{c}\text { \# de zonas afectadas } \\
\text { Una (1) }\end{array}$ & $\begin{array}{c}\text { Edema } \\
\text { Localizado a la zona (1) }\end{array}$ & $\begin{array}{c}\text { Fase de cicatrización } \\
\text { Granulación (2) }\end{array}$ \\
\hline \multicolumn{2}{|c|}{ Neuropatia } \\
Inicial (1)
\end{tabular}

\section{- Según clasificación PEDIS:}

\begin{tabular}{|l|c|c|}
\hline \multicolumn{1}{|c|}{ Evidencia clínica } & Gravedad & PEDIS \\
\hline $\begin{array}{l}\text { Herida que presentaba eritema menor de } \\
\text { 2cm, en los márgenes de la lesión rubor, } \\
\text { supuración y tejido de granulación. }\end{array}$ & Leve & Tipo 2 \\
\hline
\end{tabular}

• Según la clasificación Wagner:
\begin{tabular}{|c|c|c|}
\hline Caracteristicas & Lesión & Grado \\
\hline Destrucción del espesor total de la piel. & $\begin{array}{c}\text { Úlcera } \\
\text { superficial }\end{array}$ & I \\
\hline
\end{tabular}


Tabla 2. Planteamientos de los procesos de atención

\begin{tabular}{|c|c|c|c|}
\hline Valoración & Diagnóstico nanda & Planeación - NOC & Ejecución - intervenciones NIC \\
\hline $\begin{array}{l}\text { Datos objetivos: úlceras } \\
\text { superficiales como complicación } \\
\text { del pie diabético, eritema menor } \\
\text { de } 2 \mathrm{~cm} \text { en los márgenes, rubor, } \\
\text { supuración y tejido de } \\
\text { granulación. }\end{array}$ & $\begin{array}{l}\text { Deterioro de la integridad } \\
\text { cutánea R/C factores externos } \\
\text { (presión) y factores internos } \\
\text { (alteraciones del metabolismo y } \\
\text { de la sensibilidad). M/P } \\
\text { alteración de la integridad } \\
\text { cutánea. }\end{array}$ & $\begin{array}{l}1101 \text { Integridad tisular: piel y } \\
\text { membranas mucosas. } \\
1103 \text { Curación de la herida: por } \\
\text { segunda intención. } \\
0204 \text { Consecuencias de la } \\
\text { inmovilidad: fisiológicas. } \\
0917 \text { Estado neurológico: periférico. } \\
2400 \text { Función sensitiva: táctil. } \\
0407 \text { Perfusión tisular: periférica. }\end{array}$ & $\begin{array}{l}3660 \text { Cuidado de las heridas. } \\
3590 \text { Vigilancia de la piel. } \\
1660 \text { Cuidados de los pies. } \\
\mathbf{5 6 0 3} \text { Enseñanza: cuidados de los pies. } \\
\mathbf{3 4 8 0} \text { Monitorización de las } \\
\text { extremidades inferiores. } \\
\mathbf{6 5 4 0} \text { Control de infecciones. }\end{array}$ \\
\hline
\end{tabular}

- El enfermero monitorizará y documentará las características de la herida: localización, tamaño, aspecto, tipo de drenaje, color, tamaño y olor para llevar control de cambios y avances que se presentan en la misma.

- Colocará un vendaje apropiado para la herida para evitar infecciones y favorecer procesos de cicatrización y recuperación cutánea.

- Cambiará los apósitos y vendajes de acuerdo con la cantidad de exudado y drenaje para evitar procesos de infección e inspeccionará la herida para llevar control de los cambios cutáneos.

- Colocará mecanismos de alivio de presión (colchones con baja perdida de aire, almohadillas, cojines o apósitos liberadores de presión) en los pies del paciente ya que estos son los que presentan mayor alteración para evitar deterioro de la integridad tisular.

- Ayudará al paciente y su familia a obtener los materiales necesarios para favorecer los procesos de recuperación en caso de que estos no cuenten con los medios adecuados (con servicios sociales).

- Enseñará al paciente y su familia a reconocer los signos/síntomas de infección para evitar complicaciones en el proceso de cierre/cicatrización de la herida.

- Enseñará al paciente y su familia los procedimientos de limpieza, curación y cuidado de la herida que se deben realizar posterior al egreso del paciente, así como la utilización, el almacenamiento y el correcto desecho de los apósitos y el material de cura que se puede llegar a utilizar.

- Enseñará al paciente y familia la importancia del cuidado de los pies debido al diagnóstico de diabetes y sus implicaciones vasculares y nerviosas.

- Enseñará al paciente y familia la importancia de inspeccionar el calzado y de elegir el mismo de forma correcta para evitar complicaciones vasculares periféricas y procesos de infección.

- Enseñará al paciente y familia a vigilar la temperatura de los pies con el dorso de la mano y a inspeccionar el estado de éstos (por ejemplo, utilizando un espejo que tenga aumento ya que el paciente presenta disminución de la visión) así mismo como el estado de las uñas, su coloración y los espacios interdigitales.

- Valorará los reflejos tendinosos profundos, la percepción de vibración, el grado de sensibilidad y las características de los pulsos pedio y tibial para reconocer el grado de afectación nerviosa y vascular.

- Enseñará al paciente y familia la importancia del lavado de manos y su correcta forma de realización antes y después de manipular las heridas y zonas por presión para evitar procesos infecciosos.

Datos objetivos: paciente que en su estancia hospitalaria inicial no tenía familiares ni acompañantes y vivía solo.

Datos subjetivos: refería: “...no soy amiguero ni nada de esas cosas.../prefiero que nadie sepa que estoy acá...".

\section{Procesos familiares} disfuncionales $\mathrm{R} / \mathrm{C}$ estrategias de afrontamiento ineficaces $M / P$ deterioro de la comunicación y la dinámica familiar.
2602 Funcionamiento de la familia.

2603 Integridad de la familia.

2600 Afrontamiento de los

problemas de la familia.

2609 Apoyo familiar durante el tratamiento.

0902 Comunicación.

2608 Resiliencia familiar.
4360 Modificación de la conducta.

5230 Mejorar el afrontamiento.

5602 Enseñanza: proceso de enfermedad.

7130 Mantenimiento de procesos

familiares.

8340 Fomentar la resiliencia.

4410 Establecimiento de objetivo comunes.

5270 Apoyo emocional.

7140 Apoyo a la familia.

7100 Estimulación de la integridad familiar.

- El enfermero valorará la reacción emocional de la familia frente a la enfermedad del paciente para reconocer las necesidades ante el diagnóstico, pronóstico y presentación de complicaciones (como el pie diabético), entre otros.

- Favorecerá una relación de confianza con la familia para mejorar la comunicación e informarlos sobre el estado del paciente.

- Generará un ambiente terapéutico y fomentará el apoyo familiar durante la estancia hospitalaria para la recuperación del paciente.

- Responderá todas las preguntas que tenga tanto el paciente como su familia y escuchará las preocupaciones y sentimientos de los mismos para favorecer la integración familiar.

- Proporcionará ayuda para cubrir las necesidades básicas de la familia (por ejemplo, direccionando el caso a servicios sociales) si éstos no cuentan con los recursos apropiados.

- Ayudará a identificar las fortalezas y oportunidades de mejora en la familia (en términos de comunicación, de tiempo y de apoyo) para que se puedan desarrollar actividades basadas en lo que se identifique.

- Proporcionará información de los progresos del paciente a su familia (de acuerdo con lo que éste desee y permita) para generar una actitud positiva hacia la recuperación.

- Enseñará a la familia los planes médicos y los cuidados por parte de la clínica de heridas durante la estancia hospitalaria y posterior al alta para que tanto la familia como el paciente tomen decisiones y lleguen a acuerdos sobre como los van a realizar (quienes son los cuidadores, como se van a organizar entre sí, como transportaran al paciente, quien realizará vigilancia de las heridas en el domicilio, entre otros).

- El enfermero y el personal de clínica de heridas deberá considerar la posibilidad de utilizar las TICS para realizar mayor seguimiento en el domicilio en caso de que éste no pueda asistir a citas de control externo.

- El enfermero considerará llevar el caso a servicios sociales, terapias familiares, consejeros, grupos de apoyo, atención médica domiciliaria o interconsultas por servicios psicología y geriatría si es indicado para un cuidado integral.

- Favorecerá que el paciente exprese sus sentimientos para tener una comunicación efectiva.

- Coordinará con la familia y el paciente metas en el tratamiento y se realizaran citas de revisión periódicas.

- Evaluará el nivel de conocimientos del paciente y su familia frente a la diabetes mellitus y con base en ello explicara la patología, los signos/síntomas, los factores de riesgo que dificultan el progreso de recuperación, las implicaciones vasculares, entre otros.

- Comentará la necesidad de implementar nuevos estilos de vida para evitar el progreso de la enfermedad y los cuidados que se deben brindar en el hogar (como la inspección de las heridas, las características de los pies, cambios anormales, etc...).

Fuente: Ios autores. 
Con el transcurrir de la hospitalización el paciente comenzó a presentar zonas por presión en la región plantar con eritema que no cedía ni cambiaba de características al tacto, calor local y edema, con lo cual se clasificó como una zona con cambios por presión en etapa 1 .

En general las lesiones del pie diabético intervenidas a tiempo cursan con resultados favorables utilizando los recursos y materiales adecuados. Sin embargo, el paciente tenía en ese momento diversos dispositivos que dificultaban la movilidad por sí mismo, por lo cual requirió de apoyo para evitar complicaciones clínicas, como la salida accidental de algún tubo a tórax o la descanalización intravenosa lo cual podría retrasar su recuperación. Un aspecto relevante es que no contaba con una red de apoyo familiar en el comienzo, lo cual generó dificultades en su progreso ya que los acompañantes son de gran ayuda como figuras de vigilancia y facilitan una adecuada línea de evolución, además brindan soporte emocional ya que pasa de ser una persona autónoma a alguien dependiente para realizar actividades cotidianas. El enfermero enseñará la forma de realizar los procesos de atención para obtener mejores resultados y la recuperación del paciente, como se indica en la tabla 2.

\section{CONCLUSIONES}

La diabetes mellitus (DM) es una patología de gran impacto en el mundo no solo por la prevalencia e incidencia sino por las implicaciones físicas, familiares, sociales y económicas que afectan al paciente, a sus familias por el requerimiento de un cuidado y vigilancia doméstico necesario para evitar progreso de la enfermedad y a los sistemas de salud por el uso de recursos tanto materiales como humanos, los cuales no siempre logran garantizar la supervivencia del paciente y que en ocasiones se vuelven una utopía por sus altos costos y difíciles métodos de acceso. De otro lado considero que se deben reforzar los planes y programas de prevención primaria y secundaria de esta patología ya que favorecería el reconocimiento de los factores de riesgo, así como el diagnóstico y tratamiento farmacológico tempranos y el seguimiento estricto para detectar las complicaciones (pie diabético). Si bien no es una tarea fácil, las intervenciones multidisciplinarias coordinadas son de gran aporte para dar frente a esta patología y para evitar el deterioro del estado general de los pacientes diagnosticados con DM.

Como enfermera profesional reconozco que nuestra labor va más allá de la atención asistencial de estos pacientes, dado que contamos con los conocimientos y los recursos para establecer una comunicación terapéutica efectiva entre los pacientes y sus respectivas familias, para dar educación en manejos farmacológicos, en implementación de diversos estilos de vida y en proveer información sobre la respectiva enfermedad (fisiopatología, signos, síntomas, tratamientos, entre otros). En la narrativa descrita reconocí que había necesidades físicas, pero también familiares por lo cual se creó un puente de comunicación con otros servicios que pueden establecer contacto con las personas adecuadas para favorecer el proceso de recuperación y rehabilitación. Si bien se realizaron las intervenciones físicas que requería, se aprovechó para crear un entorno familiar apropiado en donde debe primar la comunicación entre todos los miembros y se reforzaron las responsabilidades de todos los individuos (incluyendo el paciente) para evitar el progreso de la enfermedad, para llegar a las metas del tratamiento y que pueda pueda retornar a unas actividades autónomas, pero con una mejor calidad y estilo de vida.

\section{REFEREN CIAS}

1. Cubero Alpízar CM, Rojas Valenciano L. Intervenciones de enfermería para mejorar la calidad de vida de las personas con pie diabético. Journal Health NPEPS. 2018;3(2):566-82.

2. de Souza Teixeira CR, Becker TA, Citro R, Zanetti ML, Landim CA. [Validation of nursing interventions in people with diabetes mellitus]. Rev Esc Enferm USP. 2011;45(1):173-9. doi: 10.1590/ s0080-62342011000100024. Validacao de intervencoes de enfermagem em pessoas com diabetes mellitus.

3. Wild S, Roglic G, Green A, Sicree R, King H. Global prevalence of diabetes: estimates for the year 2000 and projections for 2030. Diabetes care. 2004;27(5):1047-53. doi: 10.2337/diacare.27.5.1047

4. Aalaa M, Malazy OT, Sanjari M, Peimani M, Mohajeri-Tehrani M. Nurses' role in diabetic foot prevention and care; a review. J Diabetes Metab Disord. 2012;11(1):24. doi: 10.1186/2251-6581-11-24. 